\title{
Freud in Amerika
}

\author{
Vera Saller (Zürich)
}

Zusammenfassung: 1909 reiste Sigmund Freud in Begleitung von Carl G. Jung und Sandor Ferenczi zu einer Vortragsreihe nach Amerika. In diesem Artikel wird untersucht, was die Umstände der euphorischen Aufnahme der Psychoanalyse durch amerikanische Ostküsten-Intellektuelle und wenig später durch die Öffentlichkeit waren. Anhand einer kurzen Schilderung von Freuds Gastgeber Stanley Granville Hall, James Jackson Putnam und William James wird dargestellt, wie die amerikanischen Wissenschaftler in je verschiedener Weise ambivalent zwischen naturwissenschaftlichem Reduktionismus und Transzendentalismus standen. Für die begierige Aufnahme der psychoanalytischen Therapie unter Psychiatern und Neurologen mache ich deshalb den starken Wunsch verantwortlich, dem materialistischen Weltbild zu entkommen. Zudem war ein grosses Bedürfnis da, hilfreiche Strategien für den Umgang mit Kranken zu finden. Für die weitere Entwicklung bis in die späten 50er-Jahre stelle ich in der psychotherapeutischen und institutionellen psychoanalytischen Landschaft eine starke Tendenz zur Erstarrung und Moralisierung fest. Für die Aufnahme durch die Öffentlichkeit und die tiefe Durchdringung der amerikanischen Populärkultur mit Gedanken und Schlagwörtern aus der Psychoanalyse gilt eher das Gegenteil. Ein liberales Verständnis der Psychoanalyse ermöglichte die Enttabuisierung von neurotischen, persönlichen Schwierigkeiten, die in Film und Fernsehen zunehmend thematisiert wurden. Was die Psychoanalyse indessen ebenfalls für die Medien attraktiv gemacht hat, ist der simple Grundsatz "sex sells".

Schlüsselwörter: Hall, Putnam, James, Laienanalyse, Ego-Psychologie, Standard Edition, Populärkultur

\section{Freuds Vorlesungen an der Clark University, Worcester: die Gastgeber}

Eine Vielzahl amerikanischer Autoren hat sich mit damit befasst, warum die Psychoanalyse von Amerika so euphorisch aufgenommen wurde und wie sie die amerikanische Kultur so nachhaltig beeinflussen konnte. Dem Besuch Sigmund Freuds, der 1909 bei den 20-Jahr-Feierlichkeiten der Clark-Universität im eine Autostunde von Boston entfernten Worcester eine Reihe von Vorträgen hielt und der 
dort - zusammen mit zwanzig anderen Geehrten - einen Ehrendoktortitel erhielt, wird bei diesen Betrachtungen und Forschungen allgemein eine grosse Bedeutung eingeräumt (vgl. Jones, 1984, S. 75 ff.; Hale, 1971; aber auch Skues, 2012). Der 2013 verstorbene Nathan G. Hale, dessen zweibändige Anthologie über Psychoanalyse in Amerika Standards setzte, führt aus, dass die Clark-Einladung an Freud strategisch im genau richtigen Moment erfolgt sei. Freuds Clark-Vorlesungen betonten einige Elemente seiner Theorie, die den pragmatisch gesinnten Amerikanern sehr entgegenkamen:

Freud betonte die Zweckmässigkeit, den Optimismus, die vergleichbare Einfachheit der Psychoanalyse, und er löste einige Diskussion über die Sublimation aus. (Hale, 1971, S. 5; Übersetzung: V. S.)

Er präsentierte eine Methode der Psychotherapie, die die Rolle der Heredität herunterspielte, die Moral der Zivilisation herausforderte und die die Sexualität, die Kindheit und die Rolle des Unbewussten betonte. Mit meinen Ausführungen zu den frühen Exponenten der amerikanischen Psychoanalyse möchte ich zeigen, dass die gestandenen und prominenten Ostküsten-Intellektuellen, die Freud mit Interesse aufnahmen, Antworten zu finden glaubten für die Fragen, die sie ihr Leben lang umgetrieben hatten.

Vielleicht vorab noch ein paar Worte dazu, wie der Besuch Amerikas auf Freud selber wirkte. Wie bekannt, war Freud kein Freund von Amerika. Trotzdem hat ihm die Ehrung durch seine amerikanischen Kollegen sehr viel bedeutet. Jahre später schrieb er in seiner Autobiografie:

In Europa fühlte ich mich wie geächtet, hier sah ich mich von den Besten wie ein Gleichwertiger aufgenommen. Es war wie die Verwirklichung eines unglaubwürdigen Tagtraumes, als ich in Worcester den Katheder bestieg, um meine «Fünf Vorlesungen über Psychoanalyse» abzuhalten. Die Psychoanalyse war also kein Wahngebilde mehr, sie war zu einem wertvollen Stück der Realität geworden. (ders., 1924/1999, S. 78)

Die emotionale Reaktion Freuds muss sehr stark gewesen sein, kam sie doch selbst seinem getreuen Schüler Sandor Ferenczi etwas übertrieben vor (Ferenczi, 1988, S. 247; Falzeder, 2012, S. 107; Skues, 2011, S. 78). Trotzdem hat Freud auch später kaum gute Worte für Amerika gefunden (Falzeder, 2012). Dies, obwohl die 
amerikanischen Ausgaben seiner Schriften materiell etwas abwarfen (Bernays, 1967, S. 177 ff.) und obwohl das Interesse in den intellektuellen Zentren des noch jungen Staatenbundes sehr gross war.

Freud hatte ein sehr ambivalentes Verhältnis zum Reisen. Gemäss allen Berichten ist klar, dass die Reise nach Amerika für ihn sehr anstrengend und kräfteraubend war. Dass der 53-jährige, der mit seinen Theorien lange auf Ablehnung gestossen war, in einem Terrain, das ihm so unwirtlich und wild erschien, auf Leute traf, die sich ernsthaft für seine Arbeiten interessierten, muss für ihn wie eine Erlösung gewirkt haben. Dies umso mehr, als seine Gastgeber allesamt prominente Wissenschaftler waren, 10 bis 14 Jahre älter als er und mit abgeschlossenen Karrieren an Universitäten - ganz im Gegensatz zu den Wissenschaftlern, die sich in Wien um ihn scharten, die alle 15 bis 20 Jahre jünger waren als er.

\subsection{Stanley Granville Hall}

Stanley Granville Hall war der wichtigste unserer Protagonisten, denn er hat Freud mit seiner Einladung und mit seinem Insistieren nach einer ersten Absage nach Amerika gebracht. Hall war Präsident der Clark-Universität, deren 20-jähriges Jubiläum den Anlass für Freuds Vorträge lieferte. Die Tatsache, dass diese zentrale Figur ein Geisteswissenschaftler war, ja, der Mann sogar als der Begründer der - experimentellen - Psychologie in den Staaten gilt, mag erstaunen, denn die erbitterte Gegnerschaft gegenüber Laien, d.h. Nicht-Ärzten als Therapeuten, wird als typisches Merkmal der amerikanischen Psychoanalyse angesehen.

Die Ostküsten-Intellektuellen der damaligen Zeit verbrachten einen nicht unbedeutenden Teil ihrer Studien in Europa, um von der dort aktiven Forscherszene zu lernen und das dortige Bildungswesen ${ }^{1}$ zu studieren, so auch Hall. Diese Wissenschaftler hatten sich zum Ziel gesetzt, bei ihrer Rückkehr nach Amerika die Universitäten nach europäischem Vorbild aus den Fängen der Religion zu befreien und sie zu Stätten der Forschung zu machen. Hall konnte sich diesbezüglich einen Namen machen. Er war stolz auf den Aufbau der Clark-Universität, zu jener Zeit führend in der Graduierten-Ausbildung. Er war auch ein Mann, der sich ins rechte Licht setzen konnte. So war die Feier zu den 20 Jahren Clark-Universität wohl nicht zuletzt auch als Feier seiner eigenen Verdienste gedacht. In die Geschichte eingegangen ist Hall indessen eher als der Mann, der die experimentelle Psychologie von Europa nach Amerika gebracht hat. Sein Interesse an Freud und an der Psychoanalyse beruhte in erster Linie darauf, dass Freud die Sexualität thematisierte. Hall hatte selber bereits Bücher zur Bedeutung derselben geschrieben, z. B. sein berühmtes Buch zur Adoleszenz, das 1904 erschienen war. Hall hebt in diesem 
Buch die konstitutive Bedeutung der Sexualität für die menschliche Entwicklung hervor, zitiert auch Freud, aber weniger, um Freuds Theorie zu propagieren, als um seine eigene Theorie zu stützen (Prochnik, 2006, S. 124 f.). Hall hatte bezüglich Sexualität einen sozialreformerischen Impetus. Freud war später enttäuscht von ihm, weil er nach dem Abfall von Adler weiterhin dessen Ideen aufnahm. Aber Hall wollte eine synthetische Psychologie schaffen, die die Gedanken von Wundt, Freud und Adler gleichermassen aufnahm (Rosenzweig, 1992, S. 223).

\subsection{Therapie zu Zeiten von Freuds Besuch}

Psychiater und Nervenspezialisten der Ostküsten-Staaten waren schon seit längerem unzufrieden mit dem "Somatic Style" (Hale, op. cit., S. 47-68), der streng wissenschaftlichen Psychiatrie, die lediglich Krankheiten klassifizierte und Degeneration und Heredität als Erklärungsmodelle bot. Begierig nahmen sie deshalb Hinweise darauf auf, wie Krankheiten geheilt werden könnten und probierten Therapiemodelle aus. Wenige Jahre vor Freuds Vorträgen hatte die kirchliche Emmanuel-Bewegung diesbezüglich grossen Erfolg gehabt. Die vom episkopalen Priester Elwood Worcester praktizierte Therapieform lehrte Formen der Selbstheilung und in Gruppentherapien wurden Strategien gelehrt, wie sie einige Dekaden danach auch von den Anonymen Alkoholikern verwendet wurden (McCarthy, 1984, S.93). Der Erfolg dieser Bewegung entriss der Berufsgruppe der Mediziner die Hoheit über die Krankenbehandlung, denn von psychiatrischer Seite her stand man psychischen Krankheiten noch ziemlich hilflos gegenüber. Bezüglich des Verteilkampfes um Berufsfelder hat Freud damit, dass er eine handhabbare Methode der Therapie bot, der Psychiatrie geholfen, die religiöse Vormachtstellung in der Krankenbehandlung zurückzudrängen.

Freuds Position zwischen materialistischem, biologischem und geisteswissenschaftlich-philosophischem Zugang konnte die Anhänger von experimenteller Psychologie und somatischer Neurologie durch ihre Wissenschaftlichkeit überzeugen. Es wurde aber auch der Hunger nach therapeutischer Wirksamkeit und - wie wir sehen werden - sogar derjenige nach spiritueller Nahrung gestillt durch seine frische Art, mit Symbolen umzugehen. Freud bot eine Psychologie, die sich fast ausschliesslich im Symbolischen bewegt, die aber mit dem Trieb und der Betonung der Sexualität immer auf biologisch-materielle Grundlagen verweist.

\subsection{James Jackson Putnam}

James Jackson Putnam war ein angesehener Neurologe, Angehöriger der Bostoner Oberschicht von einflussreichen Intellektuellen. Ein Zitat aus der 
Einleitung, die er für die englische Ausgabe von Freuds «Drei Abhandlungen zur Sexualtheorie» schrieb, zeigt, dass er von Freuds Wissenschaftlichkeit beeindruckt war.

[Freud] hat den Anteil der Triebe in jeder Phase des Lebens auf unglaublich eindringliche Weise herausgearbeitet [...]. Es kann nicht oft genug wiederholt werden, dass diese Entdeckungen keine fantastischen Schlussfolgerungen sind, sondern das Ergebnis von strikten, sorgfältigen Beobachtungen, die jeder, der sich genügend vorbereitet, verifizieren kann. (zit. nach Rosenzweig, op. cit., S. 204; Übersetzung: V. S.)

Folgen wir der Deutung von Putnams Urenkel George Prochnik (2006), galt Putnams Interesse nicht der Sexualität im Sinne von Halls Reformgeist, sondern war eher von Ralph Waldo Emersons ${ }^{2}$ Transzendentalismus gekennzeichnet. Putnam war in den Jahren seines Studiums in Deutschland Materialist und Reduktionist gewesen. Nach seiner Rückkehr in die Staaten suchte er mit beispiellosem Arbeitseifer von der beschreibenden Psychiatrie her nach Methoden, um seinen Kranken zu helfen (Prochnik, op. cit., S. 177-184). Er experimentierte mit Vorgehen, die er den Schriften und Erkenntnissen von Wundt, Janet und Freud entnahm. Nach und nach aber gelangte er zur Überzeugung, dass es keine Therapie ohne Verankerung in Spiritualität gebe. Seine therapeutischen Bemühungen und die Reflexion seiner eigenen Persönlichkeit hatten ihn mit der sexuellen Ätiologie der Neurosen vertraut gemacht. Unlösbar dagegen war für ihn der Konflikt, in den die asozialen Triebregungen den Neurotiker brachten. Jahrelang hatte Putnam damit gerungen, dass verdrängte sexuelle
Triebe geistige Störungen verursachten. Aber die Verwirklichung
dieser Impulse war wohl oder übel eine Verschwendung von Energie
oder - schlimmer - moralisch verwerflich und zersetzend für die
Gemeinschaft. (op. cit., S. 122; Übersetzung: V.S.)

Freuds Vorstellung der Sublimation schien nun dieses Problem zu lösen. Für Putnam, so schliesst Prochnik dieses Kapitel, war Freud nicht derjenige, der den Sex nach Amerika brachte, er war derjenige, der ihn von der Agenda nahm! Wie eine alchemistische Reaktion verwandelte sich Sexualität durch Sublimation in etwas Geistiges. Es war also ironischerweise die therapeutische Hoffnung, die 
bekanntlich Freud während seines langen Lebens immer mehr abhanden kam, die dem amerikanischen Optimismus entgegenkam (ebd.)!

Putnam lud Freud nach den Vorlesungen in Worcester ein, mit ihm einige Tage in Putnam Camp zu verbringen, einem primitiven Feriendorf, das sich einige Bostoner Intellektuelle gekauft hatten, um zu wandern und sich Gottes freier Natur zu erfreuen. Die beiden Männer blieben danach bis zu Putnams Tod (1918) in brieflichem Kontakt und tauschten sich auch über Privates aus.

\subsection{William James}

Der berühmte William James war Wegbereiter der Psychoanalyse einfach durch die simple Tatsache, dass er, trotz schlechten gesundheitlichen Zustandes, die Mühe auf sich genommen hatte, von Boston nach Worcester zu reisen, um Freud zu hören. James, Mitbegründer des Pragmatismus und Gründervater der amerikanischen Psychologie, war wie die anderen zitierten Grossen seiner Zeit stark damit beschäftigt, die neue, wissenschaftlich-deterministischeWeltsicht mit seiner Sehnsucht nach religiöser Geborgenheit und Vergeistigung zur Deckung zu bringen. Freuds Betonung derWissenschaftlichkeit als neuer, bessererWeltanschauung einerseits und sein unverfrorenes Spekulieren innerhalb einer philosophisch-psychologischen Sphäre andererseits interessierten ihn, auch wenn seine Kommentare über das Gehörte danach eher herabsetzend waren. Wie Rosenzweig expliziert, hatte Freud an dem Tag, an dem er James in der Zuhörerschaft wusste, bewusst über Träume gesprochen (1992, S. $25 \mathrm{ff}$.) in der Annahme, dass James dafür zu begeistern wäre. In einer ca. zwei Jahre nach dem Hören des Freud'schen Vortrags veröffentlichten Notiz verwies James jedoch, ohne Freud zu erwähnen, auf den spirituellen Charakter von Träumen, speziell seiner eigenen. ${ }^{3}$

Spiritismus ist ein weiterer Farbtupfer, der das Bild vervollständigt, wenn wir versuchen, uns vorzustellen, was vor 106 Jahren im Hause Halls vor sich ging, als James einen Abend mit den Besuchern aus Europa verbrachte. Das Thema war Spiritualismus, denn die Psychologen Hall und James hatten beide ein intensives Interesse an diesem Thema - auch wenn sie diametral entgegengesetzter Meinung waren (Rosenzweig, op. cit., S. 82 ff.; Prochnik, op. cit., S. 136 ff.). Freuds Stellungnahme an jenem Abend ist nicht überliefert. Interessant ist jedoch, dass es ähnliche Themen sein werden, die knapp vier Jahre später den Bruch zwischen Freud und Jung herbeiführen. Dass aber damals schon Abgrenzungen und Eifersucht im Untergrund eine Rolle spielten, wird aus der nachträglichen Beschreibung der Reise und der Umstände, die zu seiner, Jungs, Einladung geführt hatten, durch denselben klar (Rosenzweig, op. cit., S. 35). Der abtrünnige Kronprinz 
hat ja dann auch die Amerika-Einladung als Sprungbrett genützt, um später mit weiteren Vortragsreisen seine eigene Methode zu propagieren.

\section{$2 \quad$ Euphorie der 10er- und 20er-Jahre}

Die Psychoanalyse wurde trotz Freuds Bedenken (siehe auch unten) euphorisch aufgenommen, sowohl von der Fachwelt, im Besonderen von den psychotherapeutisch interessierten Neurologen, Psychiatern und Psychologen, aber auch von der Masse. Mit dazu beigetragen hat wohl, dass damals in Amerika professionelle und populäre Kultur nicht scharf getrennt waren (Hale, 1995, S. 20). Das Interesse zeichnete sich schon vor Freuds Amerika-Besuch ab, denn es gab bereits Buchbesprechungen zu verzeichnen und sogar schon psychoanalytische Arbeiten waren erschienen. Riccardo Steiner nennt für diesen Zeitraum eine Besprechung durch William James, sowie mindestens vier weitere Arbeiten (1987, S. 69 f.).

Nach dem Besuch Freuds schien das Interesse exponentiell zu wachsen: Nathan Hale hat nachgezählt, dass während des 1. Weltkrieges in Amerika dreimal mehr Artikel zur Psychoanalyse erschienen als zum hochaktuellen Shell Shock (1995, S. 20 f.). Der intellektuelle Hype, den die Psychoanalyse für Amerika bedeutete, zeigt sich auch daran, dass in einer Studie, die Steiner erwähnt, 200 Bücher zur Psychoanalyse zitiert wurden, die zwischen 1910 und 1920 erschienen sind (op. cit., S. 85). Ich habe bereits gezeigt, dass die amerikanischen Spezialisten sehr darum bemüht waren, Methoden zu finden, wie sie Kranken wirklich helfen konnten. Die massenhafte Inanspruchnahme durch Traumatisierte während und nach dem ersten Weltkrieg generierte mehr Prestige für die Psychiatrie, gefördert durch Berichte in Zeitungen und Journalen.

Wenn ich oben über den integren Putnam sagte, dass ihm die Psychoanalyse dazu diente, die Sexualität als menschlichem Antrieb ihres antisozialen Stachels zu entledigen, so kann vielleicht über die grossmütige Aufnahme der Methode durch die amerikanische Öffentlichkeit gerade das Umgekehrte gesagt werden: Nämlich dass die Psychoanalyse half, der durch die viktorianisch-puritanische Moral arg verpönten Sexualität zu ihrem Recht zu verhelfen. Nicht unwesentlich bei diesem Prozess waren die erst sukzessive zu mehr Einfluss gelangenden Massenmedien und ein grosses Interesse an Neurosen, Perversionen, Geisteskrankheiten. So schreibt Steiner über die durch Übersetzungen psychoanalytischer Arbeiten neu geschaffenen Ausdrücke der englischen Sprache, dass sie sowohl Fachleuten wie Laien erlaubten, innert kürzester Zeit die Grundzüge der freudschen Theorie zu verstehen. Die Vorträge von Abraham Brill in New York lösten Enthusiasmus aus und seien von Leuten aus allen intellektuellen Schichten besucht worden. Dass 
die Boulevard-Presse die psychoanalytische Fachsprache sofort aufgenommen hat, zeige, dass da wirklich ein Nerv getroffen worden sei, auch wenn dies alles vorerst nur sehr oberflächlich war (op. cit., S. 80).

Ein weiterer Faktor war die zu dem Zeitpunkt anlaufende Filmindustrie in Los Angeles, machten doch hier ebenso wie in der sich vergrössernden Population an Psychoanalytikern jüdische Einwanderer aus Osteuropa und Deutschland einen grossen Anteil aus. Eine dritte Neuerfindung der 20er-Jahre, die Werbung, wird auch oft in nächster Nähe zur Psychoanalyse gesehen, weil einer ihrer Begründer, Edward Bernays, ein Neffe von Freud war. Bernays, der in den Staaten aufgewachsen war, kannte Freud von den Sommerreisen seiner Mutter. Onkel und Neffe pflegten ein herzliches Verhältnis zueinander und Bernays sah es als seine Aufgabe, in Amerika für die Sache der Psychoanalyse zu werben. Unter anderem um seinem berühmten Onkel finanziell zu helfen, erbat er sich von seinem Onkel die Rechte für die Übersetzung einiger einführender Schriften in die Psychoanalyse.

Der von Bernays erfolgreich geprägte Slogan, der Zigaretten für Frauen als «Fackeln der Freiheit» bezeichnet, stammte eigentlich von Abraham Brill. Bernays suchte den New Yorker Analytiker zwecks Hintergrundrecherche für seinen Feldzug für die Tabakindustrie auf. Auf Bernays Frage, was die Frauen am Rauchen attraktiv fänden, antwortete ihm Brill:

Heute hat [...] die Emanzipation der Frau viele ihrer femininen Wünsche unterdrückt. [...] Zigaretten, die mit dem Mann identifiziert werden, werden zu Fackeln der Freiheit. (Bernays, op. cit., S. 248)

Bernays liess sich von Vogue eine Liste von Models geben, die er dazu bestellte, an der jährlichen Oster-Parade in New York am öffentlichsten Platz, das war damals die «Fifth Avenue», zu rauchen. Er inszenierte es als einen emanzipatorischen Akt und liess auch die Zeitungen von den Fackeln der Freiheit wissen (vgl. Bernays-Freud, 2004; Bernays, 1967; Held, 2009)!

\section{Die weitere institutionelle Entwicklung bis in die 50er-Jahre}

Ich habe anhand der Persönlichkeiten von Hall, Putnam und James versucht, ein Bild davon zu geben, auf welche Fragestellungen der amerikanischen Geisteslandschaft die Psychoanalyse traf. Dann habe ich einen Eindruck davon vermittelt, wie schnell und gründlich die Psychoanalyse in den Staaten Beachtung fand, vor allem auch bei der Bevölkerung. Wenn wir uns nun fragen, wie und ob sich 
die Psychoanalyse durch ihre Aufnahme in den Staaten verändert hat, geht es u. a. um Fragen der Übersetzungen, der Medikalisierung und damit des Ausschlusses der Laienanalyse sowie um die bei uns in Europa verpönte Ich-Psychologie.

\subsection{Die Laienfrage}

Abraham Brill und Ernest Jones hatten ein Jahr vor Freuds AmerikaVorlesungen diesen zum ersten Mal in Salzburg gesehen. Die beiden waren von Zürich her zum internationalen psychoanalytischen Kongress dort angereist. Danach sind sie nach Wien weiter gereist, um Freud dort zu besuchen. Beim Amerikabesuch im Jahr darauf war es Brill, der die Reisegruppe in New York empfing und beherbergte. Jones, der in Toronto weilte, gesellte sich in Worcester zur Reisegruppe.

Brill war bis 1910 der einzige Analytiker in NewYork, ja in ganz Amerika. Sein Beitrag zur Festsetzung psychoanalytischen Gedankenguts in den Staaten ist nicht zu unterschätzen. Er besorgte die ersten Freud-Übersetzungen und war jahrelang eine geschätzte Leaderfigur der "NewYork Psychoanalytic Society”, deren Gründung drei Monate vor jener der "American Psychoanalytic Association” (APsaA) durch Jones und Putnam erfolgt war. Geboren 1874 in einer österreichisch-galizischen Kleinstadt (heute Polen) war Brill mit 15 Jahren nach einem heftigen Streit mit seinem Vater alleine nach Amerika migriert und schlug sich mit Gelegenheitsarbeiten durch. Er schaffte es, vollkommen auf sich selber gestellt, das Medizinstudium an der Columbia University abzuschliessen und nach vier Jahren Praxis, während derer er sich intensiv vom Schweizer Psychiater Adolf Meyer ${ }^{4}$ beraten liess, reiste er über Paris nach Zürich, wo er die Psychoanalyse kennenlernte (vgl. Falzeder, 2014; Romm, 1996).

Wenden wir uns nun der zwischen Amerika und Europa heiss umkämpften Frage der Laienanalyse zu. Brill, der Freud sofort sympathisch war, und der von Freud trotz einiger Meinungsverschiedenheiten wie von einem Vater geliebt wurde, war eine der wichtigen Persönlichkeiten, die gegen die Laienanalyse eingestellt war. Als nach Putnams Tod Brill Präsident der APsaA wurde, übernahm diese seine ablehnende Haltung gegenüber Laien (Gifford \& Thompson, 2011, S. 7). Ich habe oben gezeigt, dass die Psychoanalyse der medizinischen Profession half, die Psychotherapie den Händen der Kirchen zu entreissen und sie wieder in ihr Hoheitsgebiet einzugemeinden. Eine andere Motivation, die Laienanalyse abzulehnen, war der Einsatz der Intellektuellen, die amerikanische Universitätslandschaft wissenschaftlicher und forschungsorientierter zu gestalten. Diesen Kampf führte sowohl der materialistisch denkende Hall, wie auch Putnam, der in seinen jungen 
Jahren ebenfalls ein engagierter Verfechter des wissenschaftlichen Materialismus gewesen war. Der Wunsch, speziell die medizinische Ausbildung zu verwissenschaftlichen, wird als Reaktion auf den Flexner-Report gesehen, der 1910 die Verhältnisse an den amerikanischen Ausbildungsstätten für Medizin stark kritisiert hatte (Hale, 1995, S. 33; Bohleber, 2011, S. 736; Makari, 2012, S. 114).

Einen wichtigen und interessanten Gedanken zur Laienfrage entnehme ich Michael Schröters Argumentation (1996), der den Grund des Konfliktes in einem «Konstruktionsfehler» der Psychoanalyse ortet. Die Psychoanalyse, wie Freud sie verstand, erhebe den Anspruch, allgemeine Psychologie zu sein, eine Grundlagenwissenschaft, die eigentlich an die Universität gehören würde. Als angewandte Psychoanalyse bezeichnete man deshalb ursprünglich sowohl die medizinische Anwendung (die Psychotherapie), wie auch die Anwendungen auf kulturelle Institutionen. Um die allgemeine Psychologie zu fördern, hat Freud jungen Interessierten wie Otto Rank und Theodor Reik empfohlen, nicht Medizin sondern Geisteswissenschaften zu studieren. Diese nicht-medizinischen Psychoanalytiker (später kam noch Hanns Sachs dazu) konnten indes ihren Lebensunterhalt langfristig durch Arbeiten in der Zeitschrift für Psychoanalyse, bei Imago und/oder im Internationalen Psychoanalytischen Verlag nicht sichern. Letztlich war es diesen Laien nur möglich, als Psychoanalytiker materiell zu überleben, indem sie mit Patienten arbeiteten.

Die Stimmung im Ausbildungsinstitut von Karl Abraham in Berlin war von Beginn an gegen die Arbeit von Laien mit Patienten eingestellt. Vollkommen dissident in der Frage aber wurden dann die Amerikaner, allen voran der von Freud so geschätzte Brill in New York (Nölleke, 2015)! Ich sehe dafür folgende Gründe: Die amerikanischen Psychoanalytiker der ersten Generation waren eklektizistisch. Ich habe oben Putnams Methoden erwähnt, der auch nach der Einführung der Psychoanalyse in den Kliniken weiter Elektroschocks angewendet hat, oder Hall, der weiterhin Adler rezipierte (Prochnik, op. cit., 186 f.; Makari, op. cit., S. 112). Dies brachte der amerikanischen Psychoanalyse von Seiten Freuds den Vorwurf ein, die Psychoanalyse zu verwässern (Freud, op. cit., S. 79). Spätere amerikanische Anhänger der Psychoanalyse waren nicht mehr persönlich an Freud gebunden und fühlten sich nicht verpflichtet, ihm in dieser Frage zu folgen. Die Psychoanalyse wurde in Amerika von einer Gruppe von Psychiatern und Neurologen aufgenommen, die einerseits stark an verbesserten Möglichkeiten der Therapie interessiert waren und die diesbezüglich der Psychoanalyse grosses Potenzial zuschrieben. Gleichzeitig war aber das Fachgebiet der Psychiatrie in einem starken Kampf um 
Privilegien und Prestige und man wollte verhindern, dass Laien-Therapeuten das neu geschaffene Image des Erfolgs zerstörten.

Die Amerikaner haben lange versucht, das Joch der Internationalen Vereinigung (IPA) abzuschütteln, die damals streng über die Ausbildung zum Beruf des Psychoanalytikers wachte. An der Konferenz von 1938 in Paris hatten sie vorgeschlagen, die Ausbildungskommission der IPA abzuschaffen; die IPA sollte nur noch dem wissenschaftlichen Austausch dienen. Das war für die Europäer zu diesem Zeitpunkt undenkbar. Jones wollte Zeit gewinnen und verschob die Abstimmung auf die nächste Sitzung. Die Amerikaner nutzten die Isolation und die faktische Nicht-Existenz der internationalen Zusammenarbeit während des zweiten Weltkrieges, um sich zu organisieren.

Die Zuwanderung von Flüchtlingen aus dem Dritten Reich liess die amerikanische Gesellschaft für Psychoanalyse anwachsen. Millet (1966, S. 561) berichtet, dass in der Zeit zwischen 1939 und 1941 in den Vereinigten Staaten sechs neue Gesellschaften entstanden sind. Dieser Autor war dabei, als sich die amerikanische Delegation 1948 mit Jones, dem Präsidenten der IPA, traf und als dieser - sehr überraschend - den Wünschen der Amerikaner nachkam. Damit hat Jones die dortige Medikalisierung der Psychoanalyse besiegelt und die IPA hat die Kontrolle über die Ausbildungen in den lokalen Gesellschaften abgegeben. Die strikte Beschränkung des Zugangs zur psychoanalytischen Ausbildung auf Mediziner wurde teilweise durch die Bildung nicht-IPA anerkannter Institute für Psychologen, Sozialarbeiter und Pädagogen durchbrochen, vor allem in New York. 1984 reichten vier PsychologInnen, eine Sammelklage gegen die APsaA und gleichzeitig gegen die IPA ein, sie klagten auf Behinderung des Wettbewerbs. Robert S. Wallerstein, der ein Buch über die Frage der Laienanalyse geschrieben hat, war damals Präsident der IPA und musste den Prozess mitführen. Die Resultate dieser Klage waren, dass die APsaA 1987 den alleinigen Zugang zur IPA verlor, was bedeutete, dass unabhängige psychologische Institute nun auch die Anerkennung der IPA erreichen konnten. Die APsaA passte ihre Statuten auch intern dahingehend an, dass sie die Zulassung nicht-medizinischer Ausbildungskandidaten zuliess, und schliesslich gab auch das Gericht den Klägern Recht. Diese Prozesse kamen 1988 zum Abschluss (Schröter, 2000; Pear, 1992).

\subsection{Ich-Psychologie}

Um die Entwicklungen der amerikanischen Psychoanalyse nachzuzeichnen, müsste mindestens ein zweiter Artikel, wenn nicht ein Buch geschrieben werden. Nur kursorisch hinweisen kann ich deshalb auf die gewaltige Integrationsleistung, 
die die junge Berufsgruppe zu bewältigen hatte, als mit der Machtergreifung Hitlers die deutschsprachige Psychoanalyse praktisch ausgemerzt wurde und die Analytiker im zuvor teils geschmähten, nun gelobten Land um Hilfe baten. Der Kulturschock war für beide Seiten erheblich. Hier waren die traumatisierten Europäer, die ihre persönliche Nähe zu Freud und ihre europäischen, zuvor gefestigten Traditionen einbringen wollten, auf der anderen Seite die um ihr Image bangende amerikanische Kaste der Psychiater und Neurologen. Den Ankömmlingen wurde indessen geholfen, auch wenn sie über kein medizinisches Diplom verfügten! Lawrence S. Kubie, der Vorsitzende des Komitees, das die APsaA am Tag des Anschlusses Österreichs zur Rettung der Verfolgten eingerichtet hatte, erkannte, dass die Konzentration vieler Spezialisten an einem Ort ökonomisch verhängnisvolle Folgen haben konnte und begann, die Voraussetzungen dafür zu sondieren, psychoanalytische Zentren an Orten zu bilden, wo es bis dahin keine gab. (Makari, op. cit., S. 114). Diese Taktik führte zu einer weiteren Verbreitung der populären Methode.

Aber heftige Auseinandersetzungen liessen sich nicht verhindern, vor allem in New York, wo man an den Sitzungen zeitweise mehr Deutsch als Englisch hörte. Einige der Einwanderer aus Berlin, die bereits Jahre vor der Machtergreifung Hitlers in die Staaten migriert waren (Franz Alexander, Karen Horney, Sandor Radó und Hanns Sachs) (Schröter, 1996, S. 1168), identifizierten sich stark mit den Amerikanern und übernahmen deren Positionen. Dagegen hingen vor allem die Wiener Analytiker traditionellen Vorstellungen nach und wachten streng über die Treue zum Meister. Makari stellt fest, dass sich die Situation nach 1945, nach dem Austritt der Dissidenten Karen Horney, Harry Stack Sullivan und Erich Fromm, stabilisierte. Heinz Hartmann, der neue Präsident der Vereinigung von New York, hatte einen «Stammbaum», der auf Freud zurückging. Zusammen mit seinen Kollegen aus der Wiener Zeit, Ernst Kris und Rudolph Loewenstein, gab er die theoretische Richtung für die kommenden Dekaden vor (Makari, op. cit., S. 112f.). Die Ich-Psychologie versuchte, Freuds grosse Ambition, eine allgemeine Psychologie zu schaffen, die das Normale und das Pathologische gleichermassen beschreibt, umzusetzen. Sie setzte deshalb den Schwerpunkt auf Probleme, die die Psychoanalyse mit der akademischen Psychologie ins Gespräch brachten: Wahrnehmung und Denken. Sie lehnte die Triebfreundlichkeit früherer amerikanischer Psychoanalytiker ab und warnte vor der Gefährlichkeit und Sozialschädlichkeit der Triebe - man näherte sich wieder der moralischen Position von Putnam! So etwa kommentierte Heinz Hartmann die liberalen Tendenzen einiger seiner Kollegen, man habe sich «gefährlich dem Zeitgeist genähert, der 
die Triebe feiert und nur Verachtung übrig hat für die Rationalität» (zit. nach Hale, 1995, S. 233).

Die Vorgänge stark vergröbernd könnte man sagen, dass ca. ein bis zwei Dekaden vor den angesprochenen institutionellen Veränderungen, die die Isolierung der APsaA beendeten, auch die theoretische Isolation zu bröckeln begann, weil das Interesse an den europäischen Varianten der Psychoanalyse zu wachsen begann.

\subsection{Die Übersetzung der Standard Edition}

Es war ebenfalls in den 80er-Jahren, als Kritik an der englischen Übersetzung der Schriften Freuds laut wurde. Bettelheim (1984) monierte, dass es James Strachey, dem Übersetzer der Standard Edition, nicht gelungen sei, die philosophischen Hintergrundannahmen, die das Freud'sche Weltbild prägten und seine tiefe Verankerung in der europäischen Kultur mitzutransportieren. Ganze Generationen von Analytikern hätten durch die übersetzte Standardausgabe eine Fassung der Psychoanalyse rezipiert, die die Wissenschaftlichkeit der Methode unbotmässig betonte. Bettelheim führt aus, dass Freuds Neuschöpfungen von Begriffen oft dem Alltag der deutschen Sprache entnommen waren. In der Übersetzung wurden sie fast durchwegs durch griechische oder lateinische Neologismen ersetzt, die dem Ganzen einen wissenschaftlichen Anstrich geben sollten. So wurde aus Fehlleistung parapraxis und aus Besetzung cathexis. Die amerikanische Psychoanalyse lehne sich - weit stärker als dies bei Freud der Fall war - an die Naturwissenschaften an und werde der lebendigen Seele der Menschen nicht gerecht (vgl. auch Kuenkamp, 1994; Roudinesco \& Plon, 2004, S. 980).

Dies ist insofern erstaunlich, als im Gerangel um die richtige Übersetzung letztlich London mit dem Übersetzer Strachey auftrumpfte, dessen Qualifikation in seiner Zugehörigkeit zur literarischen Szene der Bloomsbury Gruppe lag; was eher erwarten lassen würde, dass er Zwischentöne hört und Hintergründe mitübersetzt! Riccardo Steiner hat in zwei ausgezeichnet recherchierten Artikeln $(1987,1991)$ die oben angesprochenen Vorwürfe gegen Stracheys Freud-Übersetzung relativiert. Er hat die historischen Hintergründe recherchiert und die verschiedenen Strategien aufgezeigt, mit denen Freud in den englischen Sprachraum transferiert wurde. Zum Zeitpunkt, als Strachey die Gesamtübersetzung in Angriff nahm (sie erschien zwischen 1953 und 1966), war die Art der Übersetzungen vom Deutschen ins Englische schon weitgehend «standardisiert». Die Standardübersetzung, die endgültig mit Ambiguitäten aufräumen und zukünftigen Forschern einen eindeutigen, klar wissenschaftlichen Begriffsapparat zurVerfügung stellen sollte, führte die 
bereits geschaffenen Tatsachen nur noch im Detail aus. Die grosse graue Eminenz, der die szientistische Lesart von Freud anzulasten ist, wenn denn eine solche historische Aufarbeitung eine Schuldzuweisung überhaupt erlaubt, ist demgemäss Jones, und nicht Strachey.

Gehen wir nochmals zurück ins Jahr 1908. Die beiden Exponenten Jones und Brill, Psychoanalyse-Botschafter der ersten Stunde im englischsprachigen Raum, lernten Freud persönlich kennen. Zurück über den Teich, scheinen sie sich, als Brill anfing, Freud ins Englische zu übertragen und Jones in Kanada arbeitete und selber psychoanalytische Essays verfasste, gemeinsam Gedanken gemacht zu haben über die Freud'schen termini technici auf Englisch. Später machte Jones Brill das Recht auf die Übersetzung der Freud'schen Werke streitig und äusserte sich abfällig über seine Übersetzungen (Jones, op. cit., S. 64). Dass das Englisch des self-made-Mannes Brill nicht perfekt war, ist glaubhaft. Freud vermutete aber hinter den kritischen Bemerkungen von Jones Eifersucht als Motiv. Brill war, anders als Jones, aus einem ähnlichen Milieu wie Freud. Jones wurde als Nichtjude in gewissem Sinne zwar zum Nachfolger von Jung, er wurde zu «Freuds alter ego» (vgl. Brome, 1982) im englischsprachigen Kulturraum. Er wurde aber nicht mit der intensiven Freundschaft, ja der Liebe des Meisters belohnt wie Jung seinerzeit. Jones machte den Mangel an Nähe zu Freud mit seinem Talent, zu organisieren und Gruppen trotz starker innerer Widersprüche zusammenzuhalten, wett.

Jones hat offenbar ganz bewusst die Ambiguität, die gewissen freudschen Formulierungen eigen ist, auszumerzen versucht. Aufschlussreich war das Zitat, das Steiner Jones' “Papers on Psychology” entnimmt, wo Letzterer Freud dafür lobt, dass er das Geistige auf das Biologische reduziere (vgl. Steiner, 1987, S. 53, Hervorhebung V.S.). Der Reduktionismus, der sich in die Psychoanalyse Freuds durch die Übersetzung eingeschlichen zu haben schien, ist also kein zufälliges Produkt, sondern war durchaus Jones' Programm. Steiner macht darauf aufmerksam, dass Freud selber in der deutschen Fassung Mischwörter aus alten Sprachen gebraucht habe, und dass er die Neologismen aus antiken Sprachen für die Übertragung ins Englische akzeptiert zu haben scheint. Aus dem Briefwechsel zwischen Jones und Freud geht hervor, dass die beiden die Eroberung Amerikas als eine Art Verschwörung verstanden. Die Sprache, die Brill und Jones benützten, sollte von aussen besehen einfach wirken; wie im Innern eines trojanischen Pferdes, der wissenschaftlichen Sprache, sollte die für Amerikaner für unverdaulich gehaltene sexuelle Ätiologie der Neurosen eingeschleust werden (Steiner, op. cit., S. 82). Jones sah sich offensichtlich als Koordinator und Anführer der Psychoanalyse im englischen Sprachraum. In einer seltsamen Mischung aus Machthunger ${ }^{5}$ und 
Anhänglichkeit an Freud, der ihn als Goi trotz vielgepriesener Treue doch immer in einer gewissen Distanz hielt, hat Jones die Fäden gezogen und dabei viele Weichen gestellt. Ein Gedanke von Steiner, der mir für den enormen Einfluss der Psychoanalyse auf die amerikanische Populärkultur wichtig scheint, ist folgender: Der Autor erwähnt beiläufig, dass die Fachterminologie der Ärzte mit komplizierten griechisch-lateinischen Ausdrücken nicht zuletzt auch dazu diente, die Kenntnisse und Überlegungen der Ärzte vom ungebildeten Volk fernzuhalten (op. cit., S. 68). Es scheint nun, dass im demokratisch gesinnten Amerika diese Abschirmung nicht gewirkt und die schwierigen Fachwörter wie etwa der freudian slip, die complexes und die neurosis vollständig ins Alltagsvokabular eingegangen sind. ${ }^{6}$

\section{$4 \quad$ To get psyched, hit the couch und shrink}

In einer äusserst vergnüglichen Studie mit dem simplen Namen "Shrink"zeichnet Lawrence Samuel die Kulturgeschichte der Psychoanalyse in den Staaten nach. Der weit verbreitete Spitzname für Psychiater ist eine Abkürzung von head shrinker. Der Psychoanalytiker wird also mit einem Krieger verglichen, der die Köpfe seiner Feinde zu Schrumpfköpfen präpariert. Samuel nennt noch verschiedene andere umgangssprachliche Ausdrücke, z. B. habe man sich in den 20er-Jahren psychen lassen (to get psyched) während man den Besuch beim Psychiater in den 50er-Jahren eher als auf die Couch auftreffen (to hit the couch) umschrieb. Die 50er-Jahre bezeichnet er als Happy 50ies für die Psychoanalyse. Ich habe Hollywood schon erwähnt. Dort war es das Normalste, einen shrinkzu haben, und in Los Angeles gab es ein Quartier, das als “Libido Lane” verulkt wurde. Die Psychoanalyse wirkte bekanntlich auch in Plots und ins Verständnis der Figuren im Film hinein. Bekannte Beispiele dafür sind Alfred Hitchcock, John Huston und natürlich Woody Allen. Highlights der Populärkultur aus den 50er-Jahren sind auch ein Comic, in dem tatsächlich ein Psychoanalytiker den farbenfrohen Helden gibt, sowie ein Album von Bob Freeman, in dem die Psychoanalyse die Hauptrolle spielt: "Songs of couch and consultation".

\section{$5 \quad$ Schluss}

Ich habe versucht, die amerikanischen Besonderheiten der Psychoanalyse von ihrer Anfangszeit bis in die 50er-Jahre zu vergegenwärtigen. Dabei dienten mir die Pole Moralität (vgl. Putnam und die Ego-Psychologie) und Triebfreundlichkeit (vgl. die Populärkultur) einerseits, sowie von Rigidität und Überschreitung andererseits als Orientierung. 
Ich möchte noch ein letztes Beispiel anführen, das einige der hervorgehobenen Punkte in einer einzigen Person, dem Prominentenanalytiker Ralph Greenson, vereint. Greenson war einerseits ein strenger Theoretiker, der ein vielrezipiertes Lehrbuch, "The technique and practice of psychoanalysis”, geschrieben hat, anderseits war er stark mit der Filmszene identifiziert. So waren seine PatientInnen Peter Lorre, Vivian Leigh, Inge Stevens, Tony Curtis, Frank Sinatra und Marilyn Monroe und die Schilderung seiner Person und seiner Erlebnisse wurde für einen Film “Captain Newman, M. D.” (Regie David Miller) verwendet. Die Vorwürfe, die Greenson in Bezug auf den tragischen Tod von Monroe gemacht werden, reissen bis heute nicht ab (Green, 2011, S. XXXII). Greenson hatte sich mit dem Produzenten Marilyns in Verbindung gesetzt und diesem versprochen, dafür zu sorgen, dass sie am Drehplatz erscheinen würde. Er lud Marilyn zu sich nach Hause ein und machte sie mit seinen Kindern bekannt, um ihr etwas zu bieten, was sie nie hatte: ein Heim (vgl. den Film Jeudy \& Schneider, 2010). Das sind Verhaltensweisen, die wir aus heutiger Sicht als hochgradig übergriffig qualifizieren würden. André Green, der das Thema kommentierte, meinte, die Amerikaner hätten eben mehr Winnicott lesen sollen, das hätte «[...] seine professionelle Haltung gewiss stark verbessert» (ebd., S. XXXVII; Übersetzung: V. S.).

Die Aufnahme von theoretischen Inhalten aus Europa, die ja dann etwas verspätet einsetzte, schlug ein neues Kapitel in der Geschichte der amerikanischen Psychoanalyse auf. Hier bleibt nun freilich kein Raum mehr, um auf dies einzugehen. Es sei nur angedeutet, dass dieses Kapitel sicher weniger lokal im Sinne unseres Heftthemas sein würde, sondern eben global. Viele der Theorien, die heute dort entwickelt werden, könnten auch hier entwickelt werden und umgekehrt. Ob das ein Verlust ist? Das Studium der Anekdoten und Geschichten aus der Vergangenheit hat mir Spass gemacht - sicher nicht zuletzt deshalb, weil wir hier in Europa vieles von dem, was ich in seiner historischen Entstehung zu verstehen versucht habe, später übernommen haben. Und, was mir auffällt: Oft ist es uns gar nicht bewusst, wie viel von Psychoanalytischem in dem, was wir als amerikanische Alltagskultur erleben, enthalten ist.

An etwas möchte ich ganz zum Schluss noch erinnern: Ein Effekt der Popularisierung der Psychoanalyse ist, dass wir gelernt haben, über uns selber als Versager zu lachen. In diesem Sinne möchte ich meinen Aufsatz ausklingen lassen mit der mädchenhaften Stimme von Katie Lee, die auf dem oben erwähnten Album neckend singt: "Shrinker man, shrinker man, put me straight if you can!" 


\section{Literatur}

Bernays, E. L. (1967). Biographie einer Idee. Die Hohe Schule der PR. Düsseldorf \& Wien: Econ Verlag.

Bernays-Freud, A. (2004). Eine Wienerin in New York. Berlin: Aufbau Verlag.

Bettelheim, B. (1984). Freud und die Seele des Menschen. Düsseldorf: Claassen.

Bohleber, W. (2011). 100 Jahre Internationale Psychoanalytische Vereinigung. Psyche, 65, 730-751.

Burnham, J. (ed.). (2012). After Freud left. Chicago: University of Chicago Press.

Brome, V. (1982). Ernest Jones: Freud's alter ego. London: Caliban Books.

Falzeder, E. (2014). Abraham A. Brill und sein Briefwechsel mit Sigmund Freud. Sigmund Freud, Digitale Edition. http://www.freud-edition.net/essayernst-falzeder-briefe-freud-brill (30.01.2015).

Falzeder, E. (2012). "A fat wad of dirty pieces of paper": Freud on America, Freud in America and America. Burnham, J. (ed.). After Freud left. (85-109). Chicago, London: The University of Chicago Press.

Ferenczi, S. (1988). Ohne Sympathie keine Heilung. Das klinische Tagebuch von 1932. Frankfurt a. M.: S. Fischer.

Freud, S. (1924/1999). «Selbstdarstellung». Gesammelte Werke XVI. S. 31-96. Frankfurt a. M.: Fischer.

Gifford, S. \& Thompson, N. (2011). A short history of the American Psychoanalytic Association 1911 to 2011. The American Psychoanalyst, 45, 2, 7-12.

Goldschmidt, G.-A. (2010). Als Freud das Meer sah: Freud und die deutsche Sprache. Frankfurt a. M.: Fischer.

Green, A. (2011). Marilyn Monroe: death of an icon. Ders. Illusions and disillusions of psychoanalytic work. S. XXIX-XXXVII. London: Karnac Books Ltd.

Hale, N. G., Jr. (1971). Freud and the Americans. The beginnings of psychoanalysis in the United States, 1876-1917. New York: Oxford University Press.

Hale, N.G., Jr. (1995). The rise and crisis of American psychoanalysis. New York, Oxford: Oxford University Press.

Held, L. (2009). Psychoanalysis Shapes Consumer Culture. Monitor on Psychology 40 , No. 11 , S. $32-34$.

Jeudy, P. \& Schneider, M. (2010). Marilyns letzte Sitzung (Film). Arte.

Jones, E. (1984). Sigmund Freud, Leben und Werk, Band 2. Jahre der Reife. 1901-1919. München: dtv.

Kuenkamp, A. (1994). Psychoanalyse ohne Seele? In H.L. Kretzenbacher \& H. Weinrich. Linguistik der Wissenschaftssprache. (S. 121-154). Berlin \& New York: De Gruyter. 
Makari G. (2012). Mitteleuropa on the Hudson: On the struggle of American Psychoanalysis after the Anschluss. In J. Burnham (ed.). After Freud left. (S. 111-124). Chicago, London: The University of Chicago Press.

McCarthy, K. (1984). Psychotherapy and religion: The Emmanuel Movement. Journal of Religion and Health, 23, No. 2, S. 92-105.

Millet, J. A. P. (1966). Psychoanalysis in the United States. In F. Alexander. et al. (eds.). Psychoanalytic Pioneers. (S. 546-596). New York: Basic Books.

Nölleke, B. (2014). Geschichte der Psychoanalyse in den USA, Psychoanalytikerinnen.

Biografisches Lexikon. http://www.psychoanalytikerinnen.de/usa geschichte.html (25.07.204).

Pear, R. (1992). M. D.'s make room for others in ranks of psychoanalysts. New York Times Archives. http://www.nytimes.com/1992/08/19/health/md-s-makeroom-for-others-in-ranks-of-psychoanalysts.html (02.02.2015).

Prochnik, G. (2006). Putnam Camp. Sigmund Freud, James Jackson Putnam, and the purpose of American psychology. New York: Other Press.

Romm, M. E. (1966). Abraham Arden Brill, 1874-1948. First American translator of Freud. In: Alexander, F. et al. (eds.). Psychoanalytic Pioneers. (S. 210-223). New York: Basic Books.

Rosenzweig, S. (1992). The historic expedition to America (1909). Freud, Jung, and Hall the King-maker. St. Louis: Rana House.

Roudinesco, E. \& Plon, M. (2004). Wörterbuch der Psychoanalyse: Namen, Länder, Werke, Begriffe. Wien: Springer Verlag.

Samuel, L. R. (2013). Shrink. Lincoln \& London: University of Nebraska Press.

Schröter, M. (2000). Wallerstein, R. S.: Lay analysis. Life inside the controversy. Hillsdale, NJ (The Analytic Press) 1998. Buchbesprechung. Psyche, 54(3), 285-290.

Schröter, M. (1996). Zur Frühgeschichte der Laienanalyse. Strukturen eines Kernkonflikts der Freud-Schule. Psyche, 50(12), 1127-1175.

Skues, R. (2012). Clark revisited: Reappraising Freud in America. In: Burnham, J. (ed.). After Freud left. (S. 49-84). Chicago, London: The University of Chicago Press.

Steiner, R. (1991). To explain our point of view to English readers in English words. Int. R. Psycho-Analysis, 18, 351-392.

Steiner, R. (1987). A world wide international trade mark of genuineness? - Some observations on the history of the English translation of the work of Sigmund Freud, focusing mainly on his technical terms. Int. R. Psycho-Analysis, 14, 33-102. 


\section{Anmerkungen}

1 Sehr oft war das Reiseziel eine Universitätsstadt in Deutschland, die Professoren Wundt, Fechner, Meynert usw. Viele dieser Intellektuellen sprachen Deutsch, das war wohl auch der Grund, warum Freud seine Vorlesungen ohne Probleme auf Deutsch halten konnte. 2 Ralph Waldo Emerson (1803-1882), US-amerikanischer Philosoph, Schriftsteller und Vertreter des Transzendentalismus. Er hatte bei den Intellektuellen Neuenglands grosses Gewicht. In «Nature», das er 1836 im Alter von 33 Jahren veröffentlichte, schildert er die Natur als Quelle göttlicher Offenbarung. Auf Emersons Grundstück in Concord (in der Nähe von Boston) baute Henry David Thoreau (1817-1862) eine Hütte, in der er ein Jahr fern der Zivilisation lebte. Seine Erfahrung beschrieb er in «Walden».

3 Saul Rosenzweig (1992) hat in einer akribischen Rekonstruktion der Tageseindrücke, unter denen William James diese kleine Arbeit verfasst haben muss, und der Tagesreste, auf die sich die dort erwähnten Träume beziehen könnten, eine entzückende kleine Liaison des alternden William James zu einer jungen Frau zu Tage gefördert. Rosenzweig war der Meinung, damit im Nachhinein doch noch gezeigt zu haben, dass James gerade mit den Träumen, die er gegen Freud - ohne diesen allerdings namentlich zu erwähnen - anführt und die seiner, James', Meinung nach die Beziehung der Träume zum Jenseits aufzeigen, letztlich Freuds Theorie recht gebe.

4 Der Schweizer Psychiater Adolf Meyer scheint mehrmals in der Geschichte der amerikanischen Psychoanalyse grossen Einfluss gehabt zu haben, ohne sich indessen direkt der Psychoanalyse zugehörig zu fühlen.

5 Vgl. auch die Geschichte der "Brunswick Square Clinic" bei Schroeter 1996, S. $1162-1164$.

$6 \quad$ Andere Aspekte des Übersetzerproblems, etwa die These, dass es Eigenheiten der deutschen Sprache seien, die Freuds Theorien begünstigten und die eine Übersetzung in andere Sprachen erschwerten (Goldschmidt, 2010; vgl. auch Kuenkamp, 1994), können hier nicht besprochen werden. 